Department for

Innovation,

Universities \&

Skills

Full-time Young Participation by Socio-Economic Class (FYPSEC)

2008 Update

$25^{\text {th }}$ June 2008 


\title{
Full-time Young Participation by Socio-Economic Class (FYPSEC): 2008 Update
}

\begin{abstract}
Summary
The Full-Time Young Participation by Socio-Economic Class (FYPSEC) measure was introduced in 2007 and shows the proportion of young people from the top three and bottom four socio-economic classes who participate for the first time in full-time higher education, together with the difference (or "gap") between these two participation rates.
\end{abstract}

The time series has been revised in line with changes to the underlying data sources (including revised Higher Education Initial Participation Rate (HEIPR) figures and revised population estimates) and updated with the figures for $2006 / 07$. The time series now covers $2002 / 03-$ 2006/07.

Following the latest revisions and updates, FYPSEC now shows that over the period 2002/03 to 2006/07, full-time young participation for the top three socio-economic classes fell from $44.1 \%$ to $39.5 \%$, while for the bottom four socio-economic classes this has increased from $17.5 \%$ to $19.0 \%$. The socio-economic class gap between these two participation rates has consequently narrowed by 6.1 percentage points.

As this measure draws from a number of data sources and therefore contains a number of weaknesses, it should be interpreted carefully. It is recommended that the figures are used to provide trend information (i.e. change over several years) rather than fixing on year-on-year changes. Drawing conclusions from figures for individual years is not advised.

\section{Introduction}

1. The 2007 Comprehensive Spending Review Public Service Agreement number 11 is the following: "Narrow the gap in educational achievement between children from low income and disadvantaged backgrounds and their peers". One of the indicators for this PSA is the Full-time Young Participation by Socio-Economic Class (FYPSEC) measure, which looks at the gap at higher education level.

2. FYPSEC was first published in a research report in $2007^{1}$. It initially covered $2002 / 03$ 2004/05. The time series was updated later in 2007 to cover 2002/03 - 2005/06.

3. FYPSEC covers English-domiciled 18-20 year old first time entrants to full-time higher education courses at UK higher education institutions and English further education colleges, who remain on their course for at least 6 months. The measure consists of three components:

- The proportion of 18-20 year olds from the top three socio-economic classes who participate for the first time in full-time higher education;

- The proportion of 18-20 year olds from the bottom four socio-economic classes who participate for the first time in full-time higher education;

- The difference, or "gap" between these two rates.

\footnotetext{
${ }^{1}$ Kathryn Kelly \& Stephen Cook, "Full-time Young Participation by Socio-Economic Class: A new widening participation measure in higher education", Department for Education and Skills Research Report RR806 (2007): http://www.dfes.gov.uk/research/data/uploadfiles/RR806.pdf
} 


\section{Latest Figures}

The FYPSEC time series is shown in Table 1:

Table 1: Overall FYPSEC figures:

\begin{tabular}{|l|r|r|r|r|r|}
\hline & $\mathbf{2 0 0 2}$ & $\mathbf{2 0 0 3}$ & $\mathbf{2 0 0 4}$ & $\mathbf{2 0 0 5}$ & $\mathbf{2 0 0 6}$ \\
\hline Participation rate for NS-SECs 1, 2, 3 & $44.1 \%$ & $40.9 \%$ & $41.2 \%$ & $42.8 \%$ & $39.5 \%$ \\
\hline Participation rate for NS-SECs 4, 5, 6, 7 & $17.5 \%$ & $17.8 \%$ & $17.4 \%$ & $19.8 \%$ & $19.0 \%$ \\
\hline Difference & $26.5 \%$ & $23.1 \%$ & $23.7 \%$ & $22.9 \%$ & $20.5 \%$ \\
\hline (Total drop in gap: 6.1 percentage points) \\
\hline
\end{tabular}

The male and female FYPSEC time series are shown in Table 2:

Table 2: Male \& Female FYPSEC figures:

\begin{tabular}{|l|r|r|r|r|r|}
\hline MALE & $\mathbf{2 0 0 2}$ & $\mathbf{2 0 0 3}$ & $\mathbf{2 0 0 4}$ & $\mathbf{2 0 0 5}$ & $\mathbf{2 0 0 6}$ \\
\hline Participation rate for NS-SECs 1, 2, 3 & $40.1 \%$ & $36.6 \%$ & $37.1 \%$ & $38.5 \%$ & $35.7 \%$ \\
\hline Participation rate for NS-SECs 4, 5, 6, 7 & $15.7 \%$ & $15.5 \%$ & $15.2 \%$ & $17.4 \%$ & $16.2 \%$ \\
\hline Difference & $24.4 \%$ & $21.1 \%$ & $21.9 \%$ & $21.1 \%$ & $19.5 \%$ \\
\hline (Total drop in gap: 4.9 percentage points) \\
\hline \multicolumn{5}{|l|}{} \\
\hline FEMALE & $\mathbf{2 0 0 2}$ & $\mathbf{2 0 0 3}$ & $\mathbf{2 0 0 4}$ & $\mathbf{2 0 0 5}$ & $\mathbf{2 0 0 6}$ \\
\hline Participation rate for NS-SECs 1, 2, 3 & $48.4 \%$ & $45.5 \%$ & $45.4 \%$ & $47.3 \%$ & $43.5 \%$ \\
\hline Participation rate for NS-SECs 4, 5, 6, 7 & $19.5 \%$ & $20.2 \%$ & $19.8 \%$ & $22.4 \%$ & $22.0 \%$ \\
\hline Difference & $28.9 \%$ & $25.4 \%$ & $25.6 \%$ & $24.9 \%$ & $21.4 \%$ \\
\hline (Total drop in gap: 7.4 percentage points)
\end{tabular}

Note: due to rounding to one decimal place, the change in gap may not appear to correspond to the gaps shown for individual years

The overall FYPSEC figures (both participation rates and their gap) are also illustrated in Figure 1 below:

Figure 1: Chart showing change in participation rates and their gap, $2002-2006$

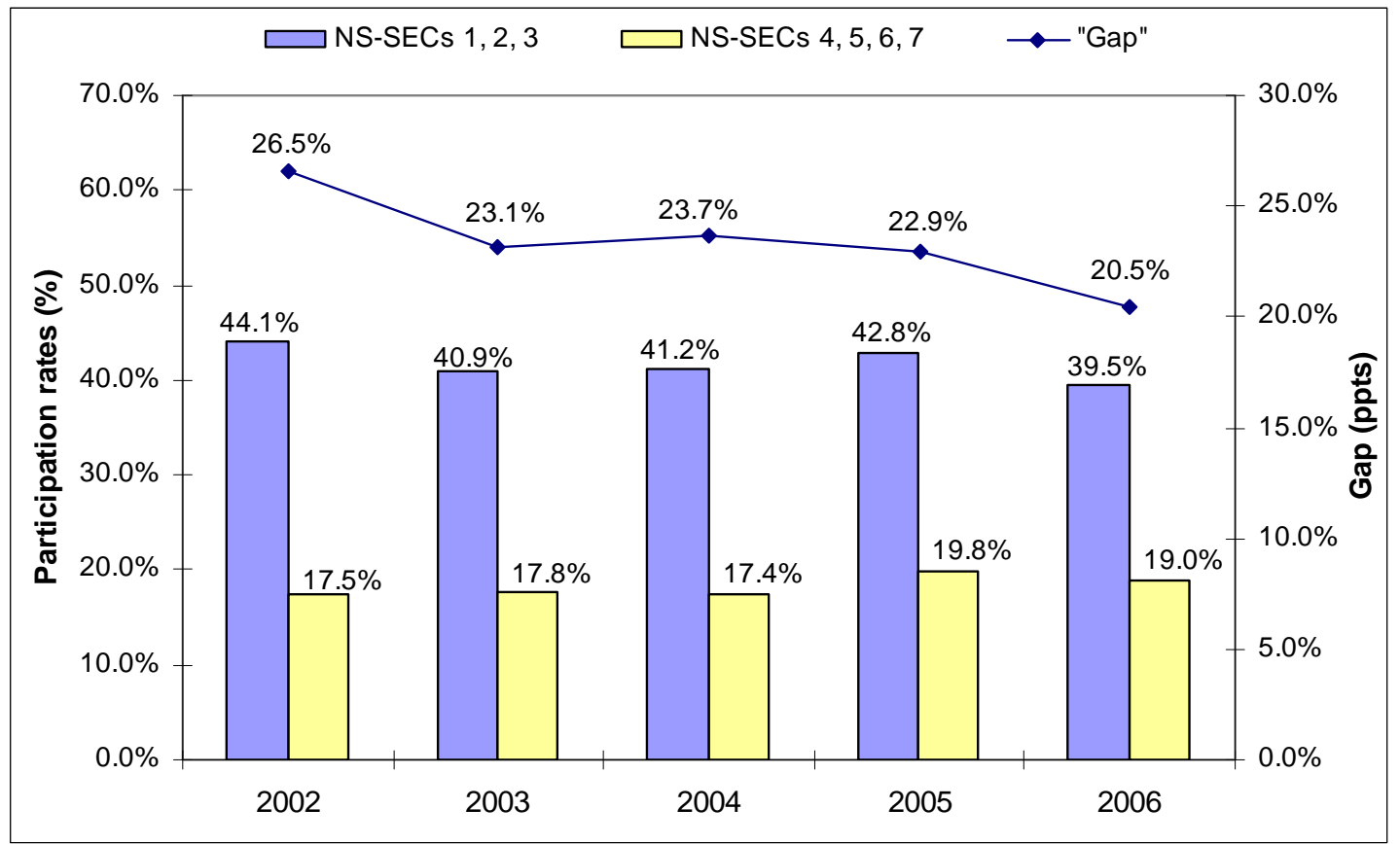




\section{Calculation details}

\section{$\underline{\text { Numerator }}$}

4. This measure is based on the students making up the 18,19 and 20 year old full-time components of the Higher Education Initial Participation Rate (HEIPR) ${ }^{2}$. Students qualifying for the HEIPR are English-domiciled first-time entrants to higher education courses, which are expected to last for at least six months, at UK Higher Education Institutions and English Further Education Colleges, and who remain on their course for at least six months.

5. The numbers of students from each socio-economic class (NS-SEC) are determined for 18 , 19 and 20 year olds separately. The students fall into three groups:

(a) Those with known NS-SEC;

(b) Those with unknown NS-SEC and known home postcode;

(c) Those with unknown NS-SEC and unknown home postcode.

6. The NS-SEC information on students in group (a) is obtained from the HESA Student Record.

7. NS-SEC information on students in group (b) is estimated using their home postcode, because there is a relationship between a person's socio-economic class and where they live. Using the home postcode an area-based measure of higher education participation level (Participation Of Local Areas, or POLAR), can be applied to each student. POLAR information is supplied by HEFCE. Initially, using only those students whose NS-SEC and home postcode are known (i.e. group (a) above), the distribution of students from each NS-SEC among the POLAR classifications is determined. The distribution of group (b) among the POLAR classifications is then determined using their home postcode, and the distribution of known NSSEC applied to these students according to their POLAR classifications. This allocates the students of group (b) among the NS-SECs in a reasonably informed way.

8. Finally, groups (a) and (b) are aggregated and the proportions of all these students who are in each NS-SEC are applied to the students of group (c). Therefore the complete unknowns are allocated to NS-SECs according to the distribution of students with known and estimated NSSEC.

9. Having obtained the number of full-time initial participants of each age, in each NS-SEC, the numbers in the top three NS-SECs are summed together, as are the numbers in the lower four NS-SECS.

\section{Denominator}

10. The NS-SEC of university entrants aged under 21 is determined by their higher-earning parent's occupation. In order to provide a comparable NS-SEC breakdown for the denominator, Labour Force Survey (LFS) socio-economic class data are used which relate to the Household Representative Persons who are parents within families with 13,14 or 15 year old children in England. The 13-15 year old proxy is applied because 18-20 year olds living with parents form a biased cohort (predominantly in full-time education).

11. The number of people in each NS-SEC is converted to a proportion by dividing the number in each NS-SEC by the total number of people with known classification. Therefore those who remain unclassified are assumed to belong to the socio-economic classes in proportion to those

\footnotetext{
2 "Participation Rates in Higher Education: Academic Years 1999/2000 - 2006/07 (Provisional)"

Statistical First Release, published by DIUS (2008)

http://www.dfes.gov.uk/rsgateway/DB/SFR/s000780/sfrdius02-2008.pdf
} 
whose NS-SEC is recorded. This is not identical to the method used in the numerator, but this assumption is made in the absence of postcode information in the available LFS household datasets.

12. The 18, 19 and 20 year old populations of England are taken from the DCSF academic year population estimates, which are based on ONS population estimates. The total NS-SEC proportions for the aggregated 13 - 15 year olds are applied to the number of 18, 19 and 20 year olds in the population of England, in order to calculate the number of people of each age, in each NS-SEC. The numbers in the top three NS-SECs are summed together, as are the numbers in the lower four NS-SECS.

\section{Calculation}

13. For each individual age, the full-time participation rate for the top three NS-SECs can be determined by dividing the number of initial entrants in the top three NS-SECs by the population in the top thee NS-SECs. The same calculation is repeated on the student and population numbers in the bottom four NS-SECs. For each group, the 18, 19 and 20 year old participation rates are summed together to provide overall initial participation rates for 18-20 year olds, one for NS-SECs 1, 2 and 3, and the other for NS-SECs 4, 5, 6 and 7.

\section{Revisions \& updates}

14. It has been necessary to make a number of revisions to FYPSEC due to changes in the underlying data. The revisions are the following:

15. FYPSEC is based on the 18,19 and 20 year old components of the Higher Education Initial Participation Rate (HEIPR), therefore any changes to HEIPR will affect FYPSEC. This year, the 2004/05 and 2005/06 HEIPR figures were both revised from a provisional status using final data on HE students in further education colleges, and so FYPSEC has been updated accordingly. This only affects part of the time series.

16. For those students whose NS-SEC is not known, for the purpose of producing FYPSEC, it is estimated using their home postcode (and subsequently HEFCE'S POLAR classification) as there is a relationship between a person's NS-SEC and where they live. As HEFCE have updated the POLAR work to reflect more recent participation levels, the calculations have been updated accordingly over the whole time series.

17. In order to estimate the socio-economic breakdown of the underlying population, we use the Labour Force Survey (LFS). This is a quarterly survey, and historically the data has covered the following quarters: Spring (March - May); Summer (June - August); Autumn (September November); Winter (December - February). From 2006 this changed to calendar quarters, i.e. Quarter 1 (January - March), Quarter 2 (April - June), Quarter 3 (July - September), Quarter 4 (October - December), and this approach has been backdated for earlier data. Originally this measure used the Spring quarter data but the calculation has been updated to use Quarter 2 data over the whole time series.

18. Following the recent revision of the ONS population estimates to more accurately account for the effects of migration, the underlying population numbers used in the FYPSEC calculation have now been updated over the whole time series.

19. As $2006 / 07$ data from all sources has become available, the time series has now been updated to include a 2006/07 figure. 


\section{Caveats}

20. The FYPSEC measure contains a number of weaknesses and as such should be interpreted carefully. It is recommended that the figures are used to provide trend information (i.e. change over several years) rather than fixing on year-on-year changes. Drawing conclusions from figures for individual years is not advised.

21. The measure draws together several data sources, including two administrative sources and sample survey data. Therefore the figures are subject to some uncertainty and volatility.

22. The measure is restricted to young full-time entrants so it only provides a partial picture of participation in higher education.

23. The use of the socio-economic class variable brings some weaknesses to the measure:

- For the young students included in this measure, socio-economic class is derived from the student's higher earning parent's occupation. This is subject to error on the part of the student (i.e. entering the wrong occupation) and on the part of the coding (i.e. wrongly classifying an ambiguous or unfamiliar occupation description).

- The proportion of students with unknown socio-economic class has been increasing over the time series. To some extent the measure accounts for unknowns by estimating socio-economic class based on postcode, as there is a relationship between a person's socio-economic class and where they live. However, while this is a more informed approach than simply assuming the unknown socio-economic class is distributed in the same way as the known socio-economic class, this is obviously still not perfect.

- Socio-economic class is derived differently for young and mature students. For those aged under 21, socio-economic class is based on the student's higher-earning parent's occupation at the time of applying. For those aged 21 and over, socio-economic class is based on the student's own occupation at the time of applying. As a result, the socioeconomic class variable is not comparable across all age ranges.

- There coverage of the socio-economic class variable is very poor for part-time students. This is because the occupation information is obtained via the UCAS application form, which is only used by applicants for full-time HE courses.

24. The 2002/03 figure includes some students who applied for entry to their courses for 2001/02 and subsequently deferred entry for one year to 2002/03. the method for coding occupation changed between $2001 / 02$ and $2002 / 03$, along with the social classification, and so these students may cause the 2002/03 figure to be slightly inaccurate.

25. The measure makes use of proxy data for the underlying population. When considering the relevant socio-economic class breakdown of the population, we look at the socio-economic class of the parents of 13, 14 and 15 year olds on the Labour Force Survey instead of parents of 18, 19 and 20 year olds. This is because the small number of 18-20 year olds who are included in the Labour Force Survey with their parents are predominantly those who are in fulltime education. Therefore a sample based on these people would be heavily biased towards those who participate in higher education. So 13-15 year olds are used as a proxy for 18-20 year olds in this case. This removes the problems of bias and small numbers but does introduce some uncertainty in that a parent's occupation (and therefore socio-economic class) could change from the time their child is 13 to the time s/he is 18.

Next update available: $2007 / 08$ figures in 2009 